\title{
Comparison of the Pharmacokinetics of Droxidopa After Dosing in the Fed Versus Fasted State and with 3-Times-Daily Dosing in Healthy Elderly Subjects
}

\author{
Jack J. Chen ${ }^{1}$ L. Arthur Hewitt ${ }^{2}$
}

Published online: 1 February 2018

(c) The Author(s) 2018. This article is an open access publication

\begin{abstract}
Background Droxidopa is an oral prodrug of norepinephrine approved for the treatment of symptomatic neurogenic orthostatic hypotension. This two-part, randomized, crossover study evaluated the 24-h pharmacokinetic profile of droxidopa in 24 healthy elderly subjects.

Methods Noncompartmental analysis was used to calculate the area under the plasma concentration-time curve (AUC), maximum plasma concentration $\left(C_{\max }\right)$, time of $C_{\max }\left(t_{\max }\right)$, and elimination half-life $\left(t_{1 / 2 \mathrm{e}}\right)$ of droxidopa and metabolites. Droxidopa was administered in the fed (highfat/high-calorie meal) or fasted state either as a single 300-mg dose (three 100-mg capsules) or 3 times/day (TID) (three 100-mg capsules) at 4-h intervals.

Results Administration of a single droxidopa dose in the fed versus fasted state decreased mean $C_{\max }$ (2057 vs $3160 \mathrm{ng} / \mathrm{mL})$ and mean AUC (10,927 vs $13,857 \mathrm{~h} \times \mathrm{ng} /$ $\mathrm{mL}$ ) and increased median $t_{\max }$ twofold (4.00 vs $2.00 \mathrm{~h}$ ). Differences between the fed and fasted state for mean $t_{1 / 2 \mathrm{e}}$ $(2.58$ vs $2.68 \mathrm{~h})$ were not observed. Fed versus fasted geometric mean ratios for $C_{\max }$ and AUC were 66\% [90\% confidence interval (CI) 60.7-71.7] and 80\% (90\% CI 72.6-88.1), respectively. With TID dosing, similar values for $C_{\max }$ were observed after each dose (range 2789-3389 $\mathrm{ng} / \mathrm{mL}$ ) with no return to baseline between doses. Norepinephrine $C_{\max }$ was $895 \mathrm{pg} / \mathrm{mL}$ following
\end{abstract}

L. Arthur Hewitt

lhew@lundbeck.com

Jack J. Chen

JChen@ketchum.edu

1 Department of Pharmacy Practice, College of Pharmacy, Marshall B. Ketchum University, Fullerton, CA, USA

2 Lundbeck, 6 Parkway North, Deerfield, IL 60015, USA dose 1, with no further increases upon subsequent doses; norepinephrine levels remained above baseline for $12-16 \mathrm{~h}$ after dose 1.

Conclusions Absorption of a single dose of droxidopa is slowed after a high-fat/high-calorie meal; for consistent effect, administer droxidopa in the same manner (with or without food). Pharmacokinetic parameters of droxidopa are similar after single and TID dosing.

ClinicalTrials.gov Identifier: NCT01149629.

\section{Key Points}

The pharmacokinetic profile of droxidopa was studied in healthy elderly volunteers.

Food effects were examined in a single-dose administration study, and the pharmacokinetic profiles of droxidopa and key metabolites associated with multiple daily dosing were determined.

Findings suggest that the absorption of a single dose of droxidopa is slowed after a high-fat/high-calorie meal [including an average $20 \%$ decrease in area under the plasma concentration-time curve (AUC) versus patients taking droxidopa under fasted conditions] and that the pharmacokinetic parameters of droxidopa are similar after single and 3-timesdaily dosing. 


\section{Introduction}

Droxidopa (L-threo-dihydroxyphenylserine) is a synthetic catecholamine prodrug that is converted to norepinephrine by dopa decarboxylase (DDC), the same enzyme that is responsible for the conversion of levodopa to dopamine (Fig. 1) [1, 2]. Droxidopa is also metabolized by catechol$O$-methyltransferase (COMT) to 3-methoxylated dihydroxyphenylserine; this is the major degradation product identified in tissue, serum, and urine [1, 2]. Norepinephrine is the key neurotransmitter responsible for mediating autonomic cardiovascular responses to orthostatic change [i.e., increases in blood pressure (BP) and heart rate]. These hemodynamic responses to orthostatic stress are required to avoid end-organ hypoperfusion, particularly that of the central nervous system, by countering the gravitationally induced shift in blood volume to the lower extremities, including the splanchnic capacitance vessels, caused by standing [1, 3].

Inadequate physiologic compensation during a positional change (i.e., supine or sitting to standing) may result in orthostatic hypotension $(\mathrm{OH})$, which is defined as a sustained BP reduction $(\geq 20 \mathrm{mmHg}$ systolic or $\geq 10 \mathrm{mmHg}$ diastolic) within $3 \mathrm{~min}$ of standing or head-up tilt [3]. Patients with neurodegenerative diseases such as Parkinson disease, multiple system atrophy (MSA), or pure autonomic failure may frequently develop neurogenic $\mathrm{OH}$ ( $\mathrm{nOH})$ because of impaired norepinephrine release associated with autonomic failure [3].

Droxidopa has been approved by the US Food and Drug Administration (FDA) for the treatment of orthostatic dizziness, lightheadedness, or the "feeling that you are about to black out" in adult patients with symptomatic nOH caused by primary autonomic failure in Parkinson disease, MSA, or pure autonomic failure; dopamine $\beta$-hydroxylase deficiency; or nondiabetic autonomic neuropathy [4]. The efficacy and safety of droxidopa have been shown in three randomized controlled trials of patients with $\mathrm{nOH}$ associated with these underlying conditions [5-8].
Previously, pharmacokinetic studies have reported droxidopa and norepinephrine levels achieved after administration of a single dose of droxidopa in patients with symptomatic $\mathrm{nOH}$ resulting from autonomic failure conditions (MSA or pure autonomic failure) [9, 10]. The current study assesses (1) the pharmacokinetic profile of droxidopa after single-dose administration, with and without food; and (2) changes in the pharmacokinetic profile of droxidopa and its metabolites (norepinephrine and 3-methoxylated dihydroxyphenylserine) associated with multiple daily dosing.

\section{Methods}

\subsection{Study Design}

The study protocol was approved by the PRACS Institute Institutional Review Board (Fargo, ND), and the study was conducted in accordance with the International Council for Harmonisation and Good Clinical Practice guidelines. Written informed consent was obtained from each subject before the initiation of study procedures. This two-part prospective study was performed to determine the pharmacokinetic profile of droxidopa and metabolites in healthy elderly subjects. In part 1 , which used a randomized crossover design, subjects received a single 300-mg dose (three 100-mg capsules) of droxidopa in either a fasted or fed state, with each state separated by a washout period (Fig. 2). In part 2, subjects returned and received 300-mg doses (three 100-mg capsules) of droxidopa three times per day (TID) (4-h intervals).

\subsection{Subjects}

Men and women (nonpregnant, nonlactating) aged $\geq 65$ years with a body mass index of $18.0-35.0 \mathrm{~kg} / \mathrm{m}^{2}$ without any clinically significant abnormal clinical findings (i.e., medical history, clinical laboratory tests, vital signs,
Fig. 1 Metabolism of droxidopa

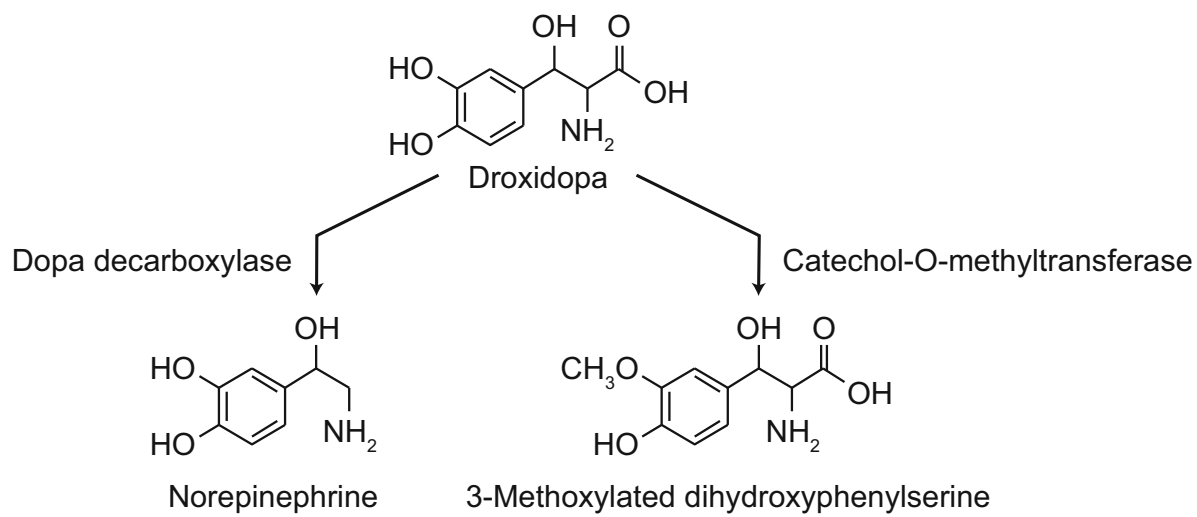



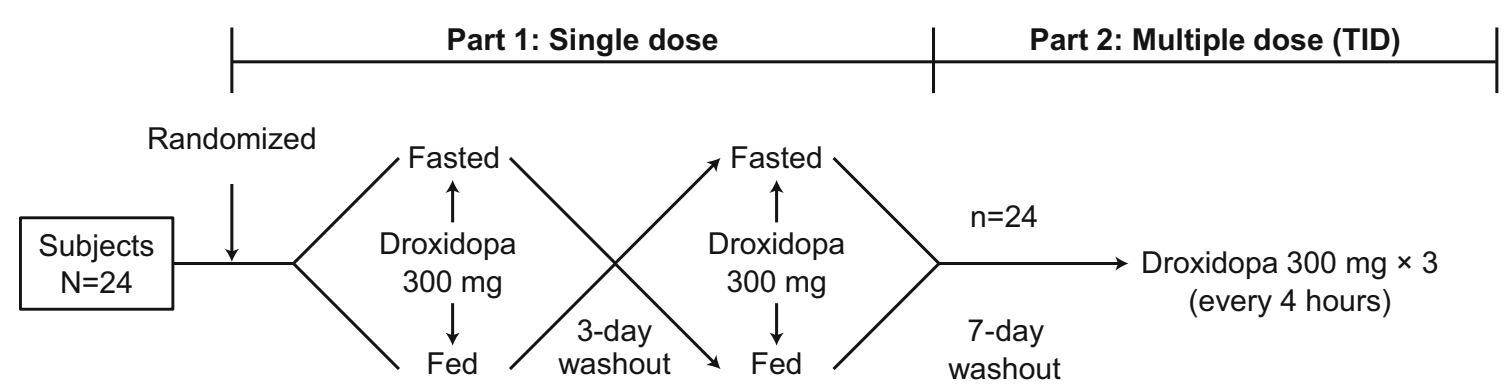

Fig. 2 Study design. TID 3 times daily

12-lead safety electrocardiogram results, or physical examination) were eligible to participate. Key exclusion criteria included presence of clinically significant cardiovascular, pulmonary, renal, endocrine, hepatic, neurologic, psychiatric, immunologic, hematologic, gastrointestinal, or metabolic disease not currently controlled with medical treatment, or presence of an active malignancy other than nonmelanomatous skin malignancies. Individuals with a history of relevant food and/or drug allergy or medication use (e.g., catecholamine-containing preparations) that could affect the pharmacokinetic or safety evaluation were also not eligible for participation.

\subsection{Treatments and Assessments}

In part 1 on the fasted dosing day, subjects received a single oral dose of three 100-mg capsules of droxidopa with water $(240 \mathrm{~mL})$ at approximately 8:00 a.m. after a minimum 10-h fast; subjects remained fasting until $4 \mathrm{~h}$ after dosing. On the fed dosing day, subjects received a standard high-calorie, high-fat breakfast at 7:30 a.m., which was to be consumed in the 30 -min period before receiving droxidopa with water $(240 \mathrm{~mL})$ at approximately 8:00 a.m. This high-calorie, high-fat meal was prepared according to specifications outlined by a guidance from the US FDA on food-effect bioavailability and fed bioequivalence studies [11] and contained 1020 calories, including $58-75 \mathrm{~g}$ of fat and 500-600 calories from fat (49-59\%). There was a 3-day washout [to allow for elimination of droxidopa based on the previously identified elimination half-life $\left(t_{1 / 2 \mathrm{e}}\right)$ ] between dosing days. Subjects remained in the study unit from the afternoon before dosing to $24 \mathrm{~h}$ after the dose of droxidopa; during this period, they were prohibited from strenuous activity. The plasma concentration of droxidopa was determined from venous blood samples taken in the semi-recumbent position after resting [10 $\mathrm{mL}$ collected in a tube containing ethylenediaminetetraacetic acid (EDTA) as a stabilizer] obtained before dosing and at $0.5,1,1.5,2,3,4,5,6,8,10,12$, and $24 \mathrm{~h}$ after dosing.
In part 2 , the same subjects returned 7 days after completion of part 1 and received three doses of droxidopa $300 \mathrm{mg}$ (three 100-mg capsules) at 4-h intervals. The first dose was administered at approximately 8:00 a.m. after an overnight fast, and the second and third doses were administered at approximately 12:00 p.m. and 4:00 p.m., respectively. Subjects were given a standard moderate-fat meal at 10:00 a.m., 2:00 p.m., and 6:00 p.m. The plasma concentrations of droxidopa, 3-methoxylated dihydroxyphenylserine, and norepinephrine were determined from venous blood samples taken in the semi-recumbent position after resting $(10 \mathrm{~mL}$ collected in a tube containing EDTA as a stabilizer) obtained before the first dose of droxidopa and at $0.5,1,1.5,2,3,4$ (before dose 2), 4.5, 5, 5.5, 6, 7, 8 (before dose 3), 8.5, 9, 9.5, 10, 11, 12, 14, 16, 18 , and $24 \mathrm{~h}$ after dosing.

In all treatment arms, water could be consumed as desired except $1 \mathrm{~h}$ before and after dosing. Caffeine-free soda and noncitrus fruit juice were allowed as desired $4 \mathrm{~h}$ after dosing treatment in part 1 and any time during part 2 except for the $2-\mathrm{h}$ periods before and after dosing.

\subsubsection{Safety}

During both parts of the study, safety was monitored by reported and observed adverse events (AEs), serial seated vital signs, resting 12-lead electrocardiograms, and assessments of clinical laboratory values and physical examination findings.

\subsection{Pharmacokinetic and Statistical Analyses}

Validated liquid chromatography tandem mass spectrometry (LC-MS/MS) methods were used to measure plasma concentrations of droxidopa, 3-methoxylated dihydroxyphenylserine, and norepinephrine. Droxidopa was extracted from dipotassium EDTA human plasma treated with reduced L-glutathione $(2.0 \mathrm{mg} / \mathrm{mL})$ and ethylene glycol-bis(2-aminoethylether)- $N, N, N^{\prime}, N^{\prime}$-tetraacetic acid (EGTA) $(1.35 \mathrm{mg} / \mathrm{mL})$ using an alumina solid phase column and perchloric acid (1 M) diluted with acetonitrile as 
the eluent; $\left[{ }^{13} \mathrm{C} 7\right]$-droxidopa was used as an internal standard. After extraction, the samples were injected into an API 4000 (SCIEX, Framingham, MA) LC-MS/MS system using a Primesep 200 column (SIELC Technologies, Wheeling IL) and a water/acetonitrile/formate mobile phase. Tandem MS monitoring was used to detect positive ions produced by the TurboIonSpray ionization source $\{\mathrm{m} /$ $z$ for droxidopa: 214.0 (parent), 152.1 (product); $\mathrm{m} / z$ for [ $\left.{ }^{13} \mathrm{C} 7\right]$-droxidopa: 221.0 (parent), 159.1 (product) .

3-Methoxylated dihydroxyphenylserine was extracted from dipotassium EDTA human plasma treated with reduced L-glutathione $(2.0 \mathrm{mg} / \mathrm{mL})$ and EGTA $(1.35 \mathrm{mg} /$ $\mathrm{mL})$ by protein precipitation with acetonitrile; $\left[{ }^{13} \mathrm{C} 7\right]-3,4-$ dihydroxyphenyl serine hydrochloride was used as the internal standard. A portion of the supernatant was transferred to a clean plate and diluted with acetonitrile. LCMS/MS and detection methods were the same as described above for droxidopa $\{\mathrm{m} / \mathrm{z}$ for 3-methoxylated dihydroxyphenylserine: 228.1 (parent), 166.2 (product); $\mathrm{m} / \mathrm{z}$ for $\left[{ }^{13} \mathrm{C} 7\right]-$ 3-methoxylated dihydroxyphenylserine: 234.1 (parent), 172.2 (product) \}.

Norepinephrine was extracted from dipotassium EDTA human plasma treated with reduced L-glutathione $(2.0 \mathrm{mg} /$ $\mathrm{mL})$ and EGTA $(1.35 \mathrm{mg} / \mathrm{mL})$ using acid-washed aluminum oxide in 96-square-well filter plates; ethylnorepinephrine was used as an internal standard. Following extraction, the analyte was eluted from the substrate using perchloric acid $(0.1 \mathrm{M})$. The eluate was then diluted with $3 \mathrm{M}$ chloroacetate buffer $(5.0 \mu \mathrm{L})$ and injected on a liquid chromatograph with an electrochemical detection system. Norepinephrine and ethylnorepinephrine were separated using an Atlantis ${ }^{\circledR} \mathrm{dC} 18$ analytical column (Waters Corporation, Milford, MA) with a mobile phase consisting of acetonitrile and chloroacetate buffer $(\mathrm{pH} \sim 3.4)$ containing sodium dodecyl sulfate and disodium EDTA; a single 3-mm glassy carbon working electrode at a potential of $+725 \mathrm{mV}$ versus a gold/gold chloride reference electrode was used for electrochemical detection.

For all pharmacokinetic analyses, actual blood sampling times were used. Noncompartmental analysis was used to measure the pharmacokinetic parameters of droxidopa (parts 1 and 2) and for the two metabolites in part 2 (3methoxylated dihydroxyphenylserine and norepinephrine).

Maximum observed plasma concentration $\left(C_{\max }\right)$ and time to achieve $C_{\max }\left(t_{\max }\right)$ were derived directly from the data. Area under the plasma concentration-time curve (AUC) to the final sample with a concentration greater than or equal to the lower limit of quantification (LLOQ) was calculated using the linear trapezoidal method. Respective LLOQs for droxidopa, norepinephrine, and 3-methoxylated dihydroxyphenylserine were $5 \mathrm{ng} / \mathrm{mL}, 50 \mathrm{pg} / \mathrm{mL}$, and $5 \mathrm{ng} / \mathrm{mL}$. Apparent terminal $t_{1 / 2 \mathrm{e}}$ was calculated from 0.693 divided by the elimination rate constant (determined from the negative slope of the terminal log-linear segment of the plasma concentration-time curve). For norepinephrine, $t_{1 / 2 \mathrm{e}}$ was not estimated because log-linear decay is not observed, as the return to endogenous levels is not constant. Geometric mean ratios and $90 \%$ confidence intervals (CIs) were determined for the fed-to-fasted ratio using the logtransformed data and the two one-sided $t$ tests procedure. All pharmacokinetic calculations and statistical analyses were performed using $\mathrm{SAS}^{\circledR}$ for Windows ${ }^{\circledR}$ version 9.1.3 (SAS Institute Inc, Cary, NC).

\section{Results}

\subsection{Demographic Characteristics}

A total of 24 subjects (79\% women) were included in the study \{mean [standard deviation (SD)] age 70.2 (4.0) years; Table 1\}. One subject vomited $3.2 \mathrm{~h}$ after dosing under a fed state in part 1. Data for this individual under the fed state were excluded from all descriptive statistics and statistical analyses. All subjects completed both parts of the study.

\subsection{Pharmacokinetic}

\subsubsection{Part 1: Fed Versus Fasted}

Administration of a single dose of droxidopa with a highfat, high-calorie meal decreased mean plasma concentration compared with dosing in the fasted state (Fig. 3). Decreases in $C_{\max }$ and AUC were observed (Table 2) after administration with food compared with the fasted state. The geometric mean ratios for $C_{\max }$ and AUC in fed versus fasted subjects were 66\% (90\% CI 60.7-71.7) and $80 \%$ (90\% CI 72.6-88.1), respectively. Administration with food increased the median $t_{\max }$ for droxidopa twofold versus the fasted state. No differences in $t_{1 / 2 \mathrm{e}}$ were noted between the fed versus fasted states. These data suggest that the differences observed for fed versus fasted

Table 1 Baseline characteristics

\begin{tabular}{lc}
\hline Variable $^{\mathrm{a}}$ & $\begin{array}{l}\text { Droxidopa } \\
(n=24)\end{array}$ \\
\hline Women, $n(\%)$ & $19(79.2)$ \\
White, $n(\%)$ & $24(100.0)$ \\
Age, years & $70.2(4.0)$ \\
Body mass index, $\mathrm{kg} / \mathrm{m}^{2}$ & $27.3(3.7)$ \\
Venous plasma norepinephrine, $\mathrm{pg} / \mathrm{mL}$ & $577(220)$ \\
\hline
\end{tabular}

$S D$ standard deviation

${ }^{\mathrm{a}}$ Values are represented as mean (SD) unless otherwise noted 
Fig. 3 Comparison of mean droxidopa plasma concentrations* for $\mathrm{fed}^{\dagger} \mathrm{vs}$ fasted administration (linear axes). *Mean (SD) concentration per time point. High-fat, high-calorie meal. $S D$ standard deviation

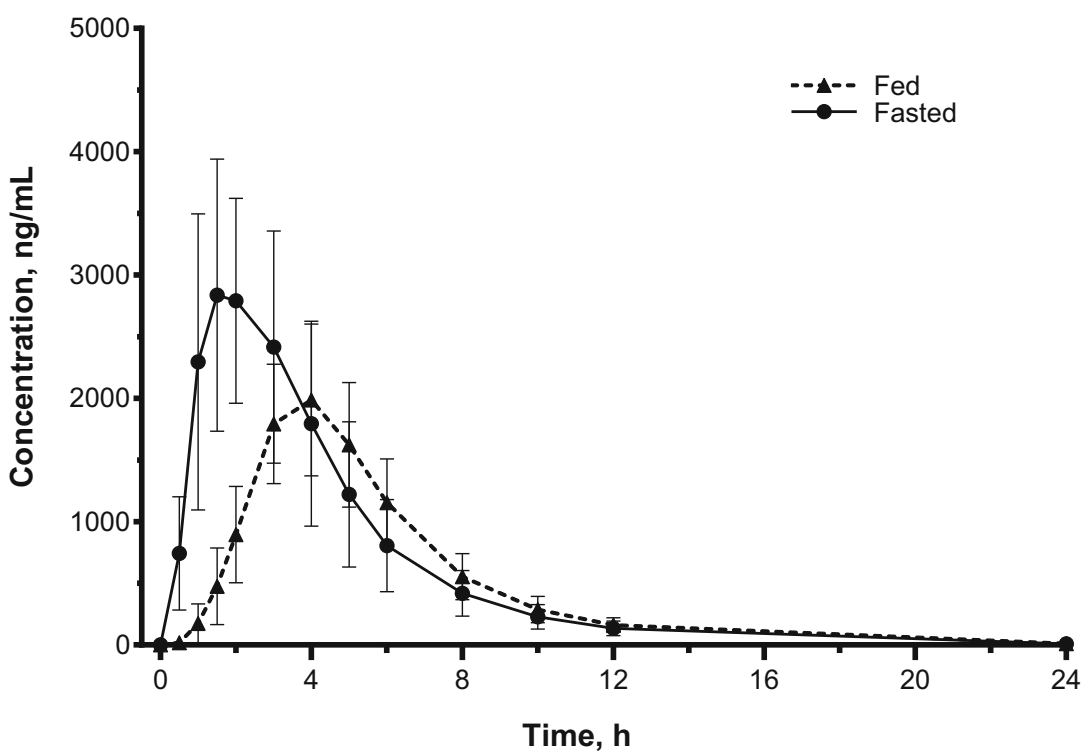

Table 2 Pharmacokinetic parameters for droxidopa (three 100-mg capsules) after fed ${ }^{\mathrm{a}}$ and fasted oral administration

\begin{tabular}{lccc}
\hline Variable & $\begin{array}{l}\text { Fed } \\
(n=23)^{\mathrm{b}}\end{array}$ & $\begin{array}{l}\text { Fasted } \\
(n=24)\end{array}$ & $\begin{array}{l}\text { Fed vs fasted } \\
\text { geometric mean ratio, \% (90\% CI) }\end{array}$ \\
\hline Mean (SD) $C_{\max }, \mathrm{ng} / \mathrm{mL}^{\mathrm{c}}$ & $2057(611)$ & $3160(1089)$ & $66.0(60.7-71.7)$ \\
Median (range) $t_{\max }, \mathrm{h}$ & $4.00(3.00-6.00)$ & $2.00(1.00-3.05)$ & ND \\
Mean (SD) AUC, $\times \mathrm{ng} / \mathrm{mL}$ & $10,927(2801)$ & $13,857(4915)$ & $80.0(72.6-88.1)$ \\
Mean (SD) $t_{1 / 2}, \mathrm{~h}$ & $2.58(0.39)$ & $2.68(0.28)$ & ND \\
\hline
\end{tabular}

$A U C$ area under the plasma concentration-time curve to the final sample with a concentration $\geq$ lower limit of quantification, $C I$ confidence interval, $C_{\max }$ peak plasma concentration, $N D$ not determined, $S D$ standard deviation, $t_{1 / 2 \mathrm{e}}$ elimination half-life, $t_{\max }$ time to maximum plasma concentration

${ }^{\text {a}}$ High-fat, high-calorie meal

${ }^{\mathrm{b}} 1$ subject vomited $3.2 \mathrm{~h}$ after dosing. Data for this individual were excluded from descriptive statistics and statistical analyses of variables

${ }^{\mathrm{c}}$ Maximum value per patient

administration of droxidopa are related to the extent of absorption and not clearance.

\subsubsection{Part 2: TID Dosing}

The plasma concentration of droxidopa over $24 \mathrm{~h}$ with TID dosing is shown in Fig. 4a. Relatively little change in the plasma concentration-time profile of droxidopa from dose to dose was observed. With TID administration of droxidopa, $C_{\max }$ of droxidopa was similar after each dose (range 2789-3389 $\mathrm{ng} / \mathrm{mL}$ ), and a between-dose return to baseline levels was not observed (Table 3). However, an increase in droxidopa plasma concentration would be expected with each dose given at 4-h intervals based on the $t_{1 / 2 \mathrm{e}}$ of approximately $2.5 \mathrm{~h}$ (found in part 1). The observed pharmacokinetic profile of droxidopa TID dosing may be attributed to food effects and the timing of dosing. The first dose was administered under fasted conditions, and the second and third doses were each administered $2 \mathrm{~h}$ after a meal (based on the 4-h dosing interval). Thus, food effects could account for the less-than-expected increases in $C_{\max }$ after the second and third doses.

Following the first dose of droxidopa, the $C_{\max }$ of norepinephrine was $895 \mathrm{pg} / \mathrm{mL}$; no further increases were observed with subsequent doses (Table 3 ). Norepinephrine levels remained above baseline for 12-16 $\mathrm{h}$ after the initial dose (Fig. 4b).

The mean plasma concentrations of 3-methoxylated dihydroxyphenylserine steadily increased after administration of the three doses of droxidopa (Fig. 4c). The $C_{\max }$ of 3-methoxylated dihydroxyphenylserine was 479, 896, and $1122 \mathrm{ng} / \mathrm{mL}$ after the first, second, and third doses of droxidopa, respectively. These findings are consistent with the mean $t_{1 / 2 \mathrm{e}}$ of $6.0 \mathrm{~h}$ (Table 3). 
Fig. 4 Mean (SD) plasma concentrations (linear axes) after droxidopa TID administration: a droxidopa, $\mathbf{b}$ norepinephrine, and $\mathbf{c}$ 3-methoxylated dihydroxyphenylserine. Arrows indicate time of dose administration. $S D$ standard deviation, TID 3 times daily
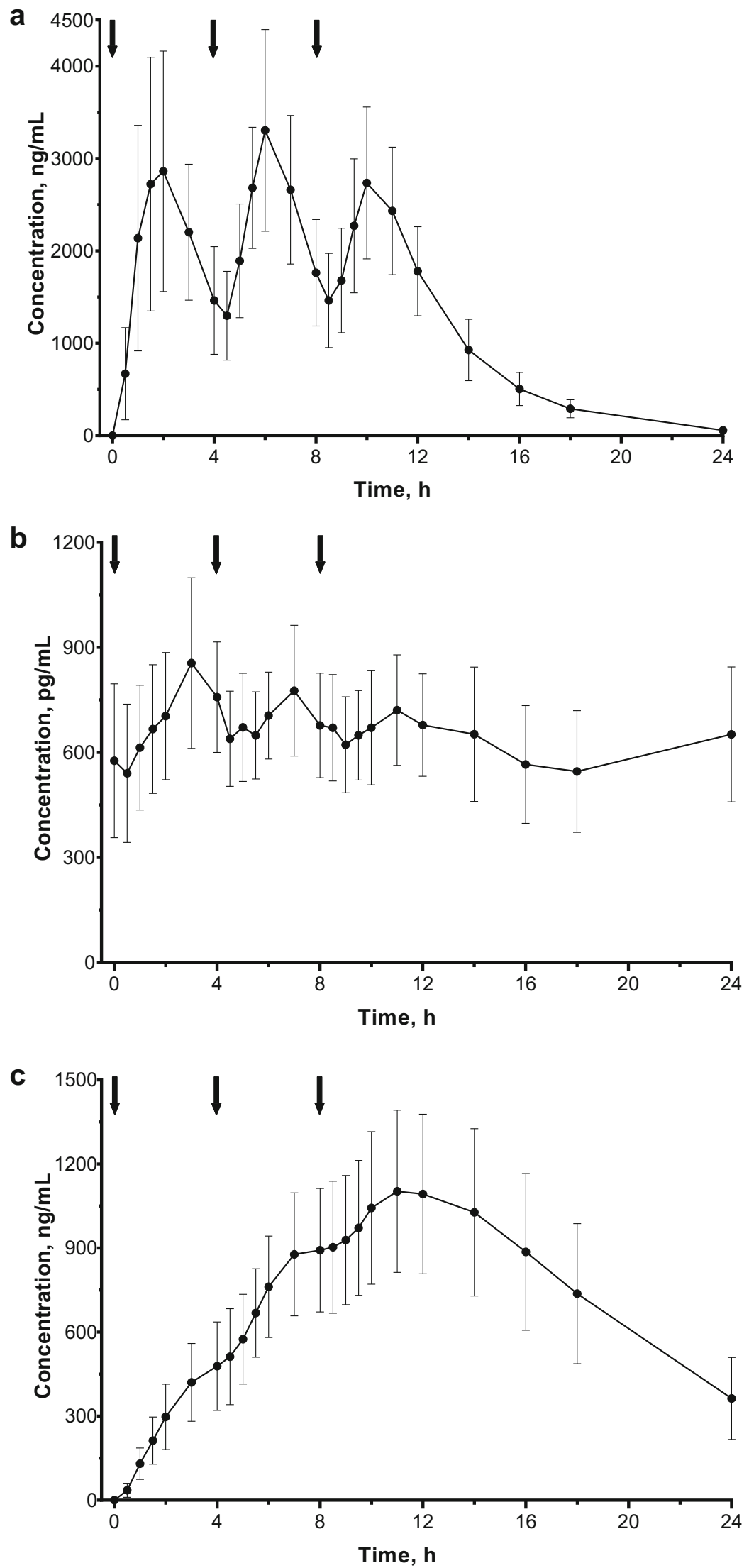
Table 3 Pharmacokinetic parameters for droxidopa, norepinephrine, and 3-methoxylated dihydroxyphenylserine with droxidopa TID dosing (three 100-mg capsules every $4 \mathrm{~h}$ )

\begin{tabular}{llll}
\hline Variable & Droxidopa & Norepinephrine & 3-Methoxylated dihydroxyphenylserine \\
\hline Dose 1 & & & \\
$\quad$ Mean (SD) $C_{\max }$ & $3113(1279) \mathrm{ng} / \mathrm{mL}$ & $895(245) \mathrm{pg} / \mathrm{mL}$ & $479(158) \mathrm{ng} / \mathrm{mL}$ \\
$\quad$ Median (range) $t_{\max }, \mathrm{h}$ & $2.00(1.00-4.00)$ & $3.00(0.00-4.00)$ & $4.00(4.00-4.00)$ \\
Dose 2 & & & $896(219) \mathrm{ng} / \mathrm{mL}$ \\
Mean (SD) $C_{\max }$ & $3389(1046) \mathrm{ng} / \mathrm{mL}$ & $840(165) \mathrm{pg} / \mathrm{mL}$ & $4.00(3.00-4.03)$ \\
Median (range) $t_{\max }, \mathrm{h}$ & $2.00(1.50-3.00)$ & $1.75(0.00-4.05)$ & $1122(286) \mathrm{ng} / \mathrm{mL}$ \\
Dose 3 & $2789(808) \mathrm{ng} / \mathrm{mL}$ & $802(160) \mathrm{pg} / \mathrm{mL}$ & $3.5(2.0-6.2)$ \\
Mean (SD) $C_{\max }$ & $2.0(1.50-3.0)$ & $3.0(0.00-16.0)$ & $16,971(4670) \mathrm{h} \times \mathrm{ng} / \mathrm{mL}$ \\
Median (range) $t_{\max }, \mathrm{h}$ & $31,648(8569) \mathrm{h} \times \mathrm{ng} / \mathrm{mL}$ & $15,601(3281) \mathrm{h} \times \mathrm{pg} / \mathrm{mL}$ & $6.01(1.04)$ \\
Mean (SD) AUC & $2.45(0.15)$ & NA & \\
Mean (SD) $t_{1 / 2 \mathrm{e}}, \mathrm{h}$ &
\end{tabular}

$A U C$ area under the plasma concentration-time curve to the final sample with a concentration $\geq$ lower limit of quantification, $C_{\max }$ peak plasma concentration, $N A$ not applicable, $S D$ standard deviation, $t_{1 / 2 \mathrm{e}}$ elimination half-life, $T I D 3$ times daily, $t_{\max }$ time to maximum plasma concentration

\subsection{Safety}

Treatment-emergent AEs (TEAEs) were reported in seven subjects (29\%; Table 4). TEAE rates were similar in parts 1 and 2. All TEAEs were mild to moderate in severity. No deaths, serious AEs, or discontinuations due to an $\mathrm{AE}$ occurred in the study. A transient mean (SD) maximum increase from baseline in systolic and diastolic BP was similar with all three dosing regimens [range 23.7-26.7
(12.0-12.6) $\mathrm{mmHg}$ and 9.6-13.5 (6.2-7.0) $\mathrm{mmHg}$, respectively]; this pharmacodynamic effect is consistent with the observed pharmacokinetic profile (i.e., maximum BP increases generally coinciding with $\left.t_{\max }\right)$. For most subjects (21/24), systolic BP remained $<150 \mathrm{mmHg}$, and the BP changes were considered clinically nonsignificant. In three subjects, transient systolic BP excursions (179, 181 , and $185 \mathrm{mmHg}$ ) were reported as TEAEs. In one of these subjects, the systolic BP excursion was measured at

Table 4 Treatment-emergent adverse events

\begin{tabular}{lll}
\hline Case & Treatment arm & Preferred term (severity) \\
\hline 1 & Single dose, fasted & Systolic blood pressure increased (mild) \\
2 & TID & Dyspepsia (mild) \\
& & Systolic blood pressure increased (mild) \\
3 & Single dose, fed & a \\
4 & Single dose, fed & Rash (mild) \\
& & Sinus headache (moderate) \\
5 & TID & Pruritus (mild) \\
7 & Single dose, fasted & Peripheral edema (mild) \\
& Single dose, fed & Systolic blood pressure increased (mild) \\
& & Nausea (mild) \\
& & Vomiting (moderate) \\
& & Balance disorder (mild) \\
& & Fall (mild) \\
& & Asthenia (mild) \\
& & Decreased appetite (mild) \\
\hline
\end{tabular}

TID 3 times daily

${ }^{\mathrm{a}} 2$ occurrences during treatment

${ }^{\mathrm{b}}$ High-fat, high-calorie meal

${ }^{\mathrm{c}}$ Occurred before TID dosing 
the pretreatment assessment before the TID dosing phase (part 2), which was after a 7-day washout period from part 1 dosing. Other than these BP excursions and symptoms listed in Table 4, no other clinically significant treatmentemergent changes in vital signs, clinical laboratory, physical examination, or electrocardiogram findings were noted.

\section{Discussion}

A high-fat, high-calorie meal slowed absorption of a single 300-mg dose of droxidopa, resulting in a twofold increase in median $t_{\max }$, a $34 \%$ decrease in mean $C_{\max }$, and a $20 \%$ decrease in mean AUC compared with fasted administration. The magnitude and onset of hemodynamic response associated with droxidopa appears to correlate with $C_{\max }$ and $t_{\max }$, respectively (rather than AUC) [10]. Therefore, administration of droxidopa with a high-fat, high-calorie meal may result in a decreased hemodynamic response. Minimal changes in the dose-to-dose plasma concentration of droxidopa were observed with TID administration. Because these findings suggest that there is a food effect on the absorption of droxidopa, it is recommended that patients always take droxidopa in the same manner, either with or without food for consistency of effects. In the phase 3 clinical trials of droxidopa, food intake was not controlled.

The pharmacokinetic parameters identified in this study of healthy subjects are generally consistent with findings from earlier pharmacokinetic studies of droxidopa performed in $\mathrm{nOH}$ populations. The droxidopa $t_{1 / 2 \mathrm{e}}$ values found in this study $(2.5-2.7 \mathrm{~h}$ in single or TID dosing regimens) are similar to values reported in a single-dose (400-mg) study in patients with $\mathrm{nOH}$ associated with pure autonomic failure or MSA (2.3-2.7 h) [9]. The droxidopa $C_{\max }$ and $t_{\max }$ values reported in this study are consistent with values previously reported. In two previous studies, a $C_{\max }$ of approximately $1900 \mathrm{ng} / \mathrm{mL}$ and $t_{\max }$ of approximately $3 \mathrm{~h}$ have been reported after single-dose administration of droxidopa (400 mg); in one study, droxidopa was administered $1 \mathrm{~h}$ after a standard breakfast [10]; in the other study, the timing of administration of droxidopa with regard to meals was not reported [9].

To the best of our knowledge, no previous published studies have reported on the systemic pharmacokinetic disposition of the droxidopa major metabolite, 3-methoxylated dihydroxyphenylserine, in human subjects. In the current study, 3-methoxylated dihydroxyphenylserine levels steadily increased after administration of the three doses of droxidopa. Although the clinical significance of this accumulation of 3-methoxylated dihydroxyphenylserine remains unknown, toxicology and safety pharmacology studies have not identified pharmacologic activity associated with this metabolite [12].

In the current study, the healthy elderly subjects had baseline venous plasma norepinephrine levels [mean (SD), $577(220) \mathrm{pg} / \mathrm{mL}$ ] within the normal physiologic range (reference range $70-1700 \mathrm{pg} / \mathrm{mL}$ depending on postural position) [13], and mean norepinephrine levels remained within the normal physiologic range (i.e., $<900 \mathrm{pg} / \mathrm{mL}$ ) during droxidopa administration every $4 \mathrm{~h}$. Although there was no nOH comparative group in this study, patients with nOH associated with MSA and Parkinson disease have physiologic venous plasma norepinephrine levels similar to those of healthy controls $[14,15]$.

Further, the healthy elderly subjects who received $300 \mathrm{mg}$ of droxidopa every $4 \mathrm{~h}$ had maximal venous plasma norepinephrine concentrations (in the semi-recumbent position) of $802-895 \mathrm{pg} / \mathrm{mL}$. These values are consistent with reported maximal venous plasma norepinephrine values between 1184 and $1250 \mathrm{pg} / \mathrm{mL}$ after a single $400-\mathrm{mg}$ dose of droxidopa in patients with $\mathrm{nOH}$ (due to pure autonomic failure or MSA) $[9,10]$. In the current study, the $t_{\max }$ of norepinephrine was approximately $1.75-3 \mathrm{~h}$ after each dose of droxidopa, despite some differences in the administration of each dose with respect to timing of meals (i.e., the first dose was administered after an overnight fast, and the second and third doses were each administered $2 \mathrm{~h}$ after a meal). This $t_{\max }$ range for norepinephrine is similar to the $3-\mathrm{h} t_{\max }$ reported after droxidopa administration in MSA patients with $\mathrm{nOH}$ (unknown timing of food ingestion with droxidopa dosing) [9].

Taken together, the single- and multiple-dose pharmacokinetic findings from this study are likely applicable to the $\mathrm{nOH}$ population and support the dosage and administration recommendations for droxidopa in the management of $\mathrm{nOH}$ [4]. Titration of droxidopa to symptomatic response is suggested because patients with $\mathrm{nOH}$ resulting from a variety of underlying autonomic failure conditions may require different maintenance doses of droxidopa based on endogenous norepinephrine levels (associated with type of neurodegeneration). A starting droxidopa dosage of $100 \mathrm{mg}$ TID is suggested, which may be increased to the maximally recommended dosage of $600 \mathrm{mg}$ TID. The findings of the current study suggest that a 300-mg TID dosing regimen does not lead to droxidopa accumulation and is associated with minimal fluctuations in norepinephrine levels.

Although this study was not designed to correlate plasma droxidopa or norepinephrine levels with hemodynamic changes, BP has been shown to rise in parallel with an increase in venous plasma norepinephrine level, with the peak BP increase preceding the peak increase in norepinephrine level [10]. Pharmacodynamically, an elevation in norepinephrine levels at night may blunt physiologic 
nocturnal BP dipping via mitigation of peripheral vasodilatation/increased systemic resistance and result in supine hypertension. Given that patients with $\mathrm{nOH}$ have circadian BP abnormalities, either as blunted nocturnal dipping or reversal of the normal circadian rhythm with higher BP values at night compared with the day, the potential effect of increased plasma norepinephrine levels must be considered [16].

However, plasma norepinephrine levels alone do not predict the occurrence of supine hypertension. The mechanism for supine hypertension in $\mathrm{nOH}$ is unclear, but appears complex and multifactorial. Various associated factors include increased vascular resistance [17, 18], elevated plasma angiotensin II levels [19], and inappropriate mineralocorticoid receptor activation [20]. In a study of patients with longstanding supine hypertension and $\mathrm{OH}$, supine resting venous plasma norepinephrine levels were in the normal range, and systemic vascular resistance (not norepinephrine levels) was associated with supine hypertension [18]. In an investigation using 24-h ambulatory BP monitoring, patients with $\mathrm{nOH}$ treated with droxidopa [mean dosage (SD) 444 (129) mg TID] experienced a nonstatistically significant increase in nocturnal mean systolic BP [7.8 (4.8) $\mathrm{mmHg} ; P=0.122]$ compared with baseline [16]. At baseline, mean nocturnal systolic BP values $\geq 160, \geq 180$, and $\geq 200 \mathrm{mmHg}$ were observed in 22,11 , and $0 \%$ of all patients, respectively; the percentages of patients with nocturnal systolic BP readings above these thresholds were similar at baseline and after a minimum of 4 weeks of droxidopa treatment [16].

In the current study, the $t_{\max }$ of norepinephrine was approximately $1.75-3 \mathrm{~h}$ after each dose of droxidopa. To maximize clinical benefit (i.e., induction of pressor effects when a patient is expected to be upright and active) and minimize risk of nocturnal supine hypertension, it is recommended that the first dose of droxidopa be taken in the morning (upon awaking) and the second dose taken midday, with the final dose taken in the late afternoon (at least $3 \mathrm{~h}$ before bedtime to avoid peak vasoconstrictor effects in parallel with $t_{\max }$ of norepinephrine).

Limitations associated with the current study include that it was conducted in healthy elderly subjects and not in patients with $\mathrm{nOH}$. Although the mean age of the subject population was typical of patients with $\mathrm{nOH}[21,22]$, other factors that may affect the pharmacokinetic findings, such as comorbid conditions or polypharmacy, were not evaluated in this study [e.g., effect of concomitant use of DDC inhibitors (e.g., carbidopa) or COMT inhibitors (e.g., entacapone)]. Because of the role of DDC and COMT in the metabolism of droxidopa (Fig. 1), administration of DDC or COMT inhibitors with droxidopa could alter pharmacokinetics and resultant pharmacodynamic and clinical effects. Studies have shown blunted BP responses and reduced norepinephrine levels when droxidopa is coadministered with carbidopa [10, 23]; however, those studies involved coadministration with a single carbidopa dose $(200 \mathrm{mg}$ ) much greater than that used in standard-ofcare clinical practice. No significant differences in the pharmacokinetic profile of droxidopa or norepinephrine were observed when droxidopa was coadministered with single-dose entacapone (200 mg) compared with droxidopa alone [23]. Integrated clinical trial data suggest that patients using a DDC inhibitor have significant improvements in orthostatic dizziness/lightheadedness symptoms (the cardinal symptom of $\mathrm{nOH}$ ), although the magnitude of the response was less when compared with non-DDC inhibitor users [24]. Thus, concomitant use of droxidopa with a DDC inhibitor may require dose adjustments. During the randomized treatment period, supine hypertension rates were similar in patients with and without concomitant DDC inhibitor use.

A further limitation of this study is that a gender subgroup analysis was not conducted for the pharmacokinetics of droxidopa. Significant sex differences in pharmacokinetics are well established [25], including for AUC of levodopa [26]. Consideration of sex differences between patients, particularly body weight differences, is thus advisable when prescribing droxidopa.

\section{Conclusions}

In summary, a high-fat, high-calorie meal slowed absorption of a single 300-mg dose of droxidopa, resulting in increased $t_{\max }$, decreased $C_{\max }$ and AUC, but similar $t_{1 / 2 \mathrm{e}}$. With TID administration, changes in the plasma concentration of droxidopa were minimal from dose to dose, and venous plasma norepinephrine levels were increased from baseline for 12-16 $\mathrm{h}$ after the first dose. The pharmacokinetic parameters (e.g., $t_{\max }, C_{\max }$, and $t_{1 / 2 \mathrm{e}}$ ) of droxidopa after single and TID dosing were similar.

\section{Compliance with Ethical Standards}

Conflict of interest Author Jack J. Chen is an employee at the Department of Pharmacy Practice, College of Pharmacy, Marshall B. Ketchum University, and provides consulting services to Lundbeck. L. Arthur Hewitt is an employee of Lundbeck.

Funding The data reported were derived from clinical trials funded by Chelsea Therapeutics, now Lundbeck. The authors received editorial assistance from CHC Group (North Wales, PA), which was supported by Lundbeck.

Ethical approval and informed consent All procedures performed in studies involving human participants were in accordance with the ethical standards of the institutional and/or national research committee and with the 1964 Helsinki declaration and its later 
amendments or comparable ethical standards. Informed consent was obtained from all individual participants included in the study.

Open Access This article is distributed under the terms of the Creative Commons Attribution-NonCommercial 4.0 International License (http://creativecommons.org/licenses/by-nc/4.0/), which permits any noncommercial use, distribution, and reproduction in any medium, provided you give appropriate credit to the original author(s) and the source, provide a link to the Creative Commons license, and indicate if changes were made.

\section{References}

1. Goldstein DS. L-dihydroxyphenylserine (L-DOPS): a norepinephrine prodrug. Cardiovasc Drug Rev. 2006;24:189-203.

2. Kaufmann H, Norcliffe-Kaufmann L, Palma JA. Droxidopa in neurogenic orthostatic hypotension. Expert Rev Cardiovasc Ther. 2015;13:875-91.

3. Freeman R, Wieling W, Axelrod FB, et al. Consensus statement on the definition of orthostatic hypotension, neurally mediated syncope and the postural tachycardia syndrome. Clin Auton Res. 2011;21:69-72.

4. Northera (droxidopa): full prescribing information. Lundbeck, Deerfield, 2016

5. Biaggioni I, Freeman R, Mathias CJ, et al. Randomized withdrawal study of patients with symptomatic neurogenic orthostatic hypotension responsive to droxidopa. Hypertension. 2015;65:101-7.

6. Hauser RA, Hewitt LA, Isaacson S. Droxidopa in patients with neurogenic orthostatic hypotension associated with Parkinson's disease (NOH306A). J Parkinsons Dis. 2014;4:57-65.

7. Hauser RA, Isaacson S, Lisk JP, et al. Droxidopa for the shortterm treatment of symptomatic neurogenic orthostatic hypotension in Parkinson's disease (NOH306B). Mov Disord. 2015;30:646-54.

8. Kaufmann H, Freeman R, Biaggioni I, et al. Droxidopa for neurogenic orthostatic hypotension: a randomized, placebo-controlled, phase 3 trial. Neurology. 2014;83:328-35.

9. Goldstein DS, Holmes C, Kaufmann H, et al. Clinical pharmacokinetics of the norepinephrine precursor L-threo-DOPS in primary chronic autonomic failure. Clin Auton Res. 2004; 14:363-8.

10. Kaufmann H, Saadia D, Voustianiouk A, et al. Norepinephrine precursor therapy in neurogenic orthostatic hypotension. Circulation. 2003;108:724-8.

11. US Department of Health and Human Services FDA. Guidance for industry: food-effect bioavailability and fed bioequivalence studies. https://www.fda.gov/downloads/drugs/guidancecompliancereg ulatoryinformation/guidances/ucm070241.pdf Accessed December 8, 2017.

12. Data on File. Deerfield. IL: Lundbeck; 2017.

13. Mayo Clinic/Mayo Medical Laboratories. Test ID CATP, catecholamine fractionation, free, plasma. http://www. mayomedicallaboratories.com/test-catalog/ Clinical+and+Interpretive/8532 Accessed March 7, 2017.

14. Goldstein DS, Holmes C, Sharabi Y, et al. Plasma levels of catechols and metanephrines in neurogenic orthostatic hypotension. Neurology. 2003;60:1327-32.

15. Sharabi $Y$, Imrich R, Holmes $C$, et al. Generalized and neurotransmitter-selective noradrenergic denervation in Parkinson's disease with orthostatic hypotension. Mov Disord. 2008;23:1725-32.

16. Kaufmann H, Norcliffe-Kaufmann L, Hewitt LA, et al. Effects of the novel norepinephrine prodrug, droxidopa, on ambulatory blood pressure in patients with neurogenic orthostatic hypotension. J Am Soc Hypertens. 2016;10:819-26.

17. Shannon JR, Jordan J, Diedrich A, et al. Sympathetically mediated hypertension in autonomic failure. Circulation. 2000;101:2710-5.

18. Schutzman J, Jaeger F, Maloney J, et al. Head-up tilt and hemodynamic changes during orthostatic hypotension in patients with supine hypertension. J Am Coll Cardiol. 1994;24:454-61.

19. Arnold AC, Okamoto LE, Gamboa A, et al. Angiotensin II, independent of plasma renin activity, contributes to the hypertension of autonomic failure. Hypertension. 2013;61:701-6.

20. Arnold AC, Okamoto LE, Gamboa A, et al. Mineralocorticoid receptor activation contributes to the supine hypertension of autonomic failure. Hypertension. 2016;67:424-9.

21. Goldstein DS, Holmes C, Sharabi Y, et al. Survival in synucleinopathies: a prospective cohort study. Neurology. 2015;85:1554-61.

22. Maule S, Milazzo V, Maule MM, et al. Mortality and prognosis in patients with neurogenic orthostatic hypotension. Funct Neurol. 2012;27:101-6.

23. Goldstein DS, Holmes C, Sewell L, et al. Effects of carbidopa and entacapone on the metabolic fate of the norepinephrine prodrug L-DOPS. J Clin Pharmacol. 2011;51:66-74.

24. Biaggioni I, Arthur Hewitt L, Rowse GJ, et al. Integrated analysis of droxidopa trials for neurogenic orthostatic hypotension. BMC Neurol. 2017;17:90.

25. Fadiran EO, Zhang L. Effects of sex differences in the pharmacokinetics of drugs and their impact on the safety of medicines in women. In: Harrison-Woolrych M, editor. Medicines for Women. Cham: Adis; 2015. p. 41-68.

26. Kumagai T, Nagayama H, Ota $T$, et al. Sex differences in the pharmacokinetics of levodopa in elderly patients with Parkinson disease. Clin Neuropharmacol. 2014;37:173-6. 